\title{
Metabolomics and inflammation in polycystic ovary syndrome
}

\author{
I. Tasci $^{1}$ (1) $\cdot$ M. I. Naharci ${ }^{1}$ (] \\ Received: 29 February 2020 / Accepted: 10 March 2020 / Published online: 19 March 2020 \\ (C) Italian Society of Endocrinology (SIE) 2020
}

Keywords Polycystic ovary syndrome $\cdot$ Metabolomics $\cdot$ Inflammation $\cdot$ Insulin resistance $\cdot$ Body composition

To the editor,

Zhang et al. [1] have successfully demonstrated some differences in the metabolic profiles in polycystic ovary syndrome (PCOS) patients based on hyperandrogenism and insulin resistance. That study also offers a panel of metabolic biomarkers to distinguish between hyperandrogenism and insulin resistance in PCOS. Thus, the results contribute to the understanding of underlying mechanisms of PCOS as well as research to improve clinical management of women with PCOS [1].

However, we noticed that the authors did not include any variable of inflammation in their study. Well conducted clinical studies have shown that women with PCOS exhibit an elevation in the level of circulating markers of inflammation that is independent of obesity [2]. A causal role for the chronic inflammation in the pathogenesis of PCOS has also been postulated based on the available data. Therefore, inflammatory variables have been suggested as a necessary part of PCOS research.

Several authors have also reached evidences of an association between certain metabolomics and chronic inflammation in PCOS patients, particularly the glycoproteins [3]. Moreover, changes in body composition parameters are more frequent in patients with PCOS and such changes as well as feeding behavior can cause some metabolites to differently modulate and/or participate in processes such as low grade inflammation.

In conclusion, we appreciate the authors' rigorous statistical performance, however, the potential of confounding of

I. Tasci

ilkertasci@yahoo.com

1 Department of Internal Medicine, Gulhane Faculty of Medicine and Gulhane Training and Research Hospital, University of Health Sciences Turkey, 06018 Ankara, Turkey the results by systemic inflammation could be acknowledged based on the available evidence.

Author contributions IT and MIN examined the study and identified the points to be addressed. IT drafted the letter and both authors read and approved the final version.

\section{Compliance with ethical standards}

Conflict of interest Authors declare that they have no conflict of interest.

Ethical approval This letter to the editor does not contain any studies with human participants or animals performed by any authors.

Informed consent Informed consent is not required in this type of article.

\section{References}

1. Zhang Z, Hong Y, Chen M, Tan N, Liu S, Nie X, Zhou W (2020) Serum metabolomics reveals metabolic profiling for women with hyperandrogenism and insulin resistance in polycystic ovary syndrome. Metabolomics 16:20. https://doi.org/10.1007/s1130 6-020-1642-y

2. Mu L, Li R, Lai Y, Zhao Y, Qiao J (2019) Adipose insulin resistance is associated with cardiovascular risk factors in polycystic ovary syndrome. J Endocrinol Invest 42:541-548. https://doi. org/10.1007/s40618-018-0949-2

3. Fuertes-Martín R, Moncayo S, Insenser M, Martínez-García MÁ, Luque-Ramírez M, Grau NA, Blanchar XC, Escobar-Morreale HF (2019) Glycoprotein A and B height-to-width ratios as obesityindependent novel biomarkers of low-grade chronic inflammation in women with polycystic ovary syndrome (PCOS). J Proteome Res 18:4038-4045. https://doi.org/10.1021/acs.jproteome.9b005 28

Publisher's Note Springer Nature remains neutral with regard to jurisdictional claims in published maps and institutional affiliations. 